\title{
The Impact of Cataract Surgery on Depression in Elderly Iranian Patients: A Case-Control Study
}

\section{Kiumars Nowroozpoor Dailami ${ }^{1}$, Mohammad Ali Heidari Gorji ${ }^{2}$, Seyed Hamzeh Hoseini $^{3}$, and Asadollah Farookhfar ${ }^{1}$}

1Department of Ophthalmology, School of Medicine, Mazandaran University of Medical Sciences, Sari, Iran

${ }^{2}$ Department of Medical-Surgical Nursing, Nasibeh School of Nursing and Midwifery, Mazandaran University of Medical Sciences, Sari, Iran

${ }^{3}$ Psychiatry and Behavioral Sciences Research Center, Addiction Institute, Mazandaran University of Medical Sciences, Sari, Iran

ORCID:

Asadollah Farookhfar: https://orcid.org/0000-0002-5584-7423

Corresponding Author: Asadollah Farookhfar; email: drfarookhfar@yahoo.com

Received 20 August 2021 Accepted 08 November 2021 Published 31 December 2021

Production and Hosting by Knowledge E

(c) Kiumars Nowroozpoor Dailami et al.. This article is distributed under the terms of the Creative Commons

Attribution License, which permits unrestricted use and redistribution provided that the original author and source are credited.

Editor-in-Chief:

Prof. Mohammad A. M. Ibnouf

\section{Abstract}

Background: Cataract-related vision impairment is clearly associated with depressive symptoms in old age. This study aimed to evaluate the effect of cataract surgery on depression among elderly Iranian patients.

Methods: In this case-control study, a total of 113 elderly patients with and without cataract who were admitted to Buali-Sina Hospital in Sari, Mazandaran, Iran were evaluated. Ophthalmological examinations were first performed at the beginning of the study and then four months later; demographic and clinical characteristics were obtained for all patients. Additionally, depression was assessed using the Hospital Anxiety and Depression Scale (HADS) for all patients during the study period.

Results: A statistically significant difference was found between the mean depression score in patients without cataract (3.28), patients with cataract surgery (3.74), and patients without cataract surgery (5.60) $(P=0.024)$. On the other hand, there was no statistically significant differences between the mean depression score in patients with cataract surgery and patients without cataract $(P=0.582)$. However, a statistically significant difference was observed between the mean depression score after cataract surgery in men (1.70) and women (4.94) $(P<0.001)$. Visual function improved in all bilateral cataract patients who had surgery in one eye. Also, the results of this study showed that in all unilateral cataract patients who had eye surgery, visual function in the left and right eyes improved.

Conclusion: We conclude that depression is more prevalent in patients with cataract compared to those without cataract. In addition, cataract surgery seems to be associated with reduced depression and better visual acuity in elderly patients with cataract.

Keywords: depressive disorder, cataract, cataract extraction, aged

\section{G OPEN ACCESS}




\section{Introduction}

Cataract is the most prevalent cause of reversible blindness worldwide. It has been estimated that cataract causes $35 \%$ of blindness and $24 \%$ of all visual impairment in the world [1, 2]. Globally, cataract surgery is one of the most prevalent surgeries, with 1.7 million surgeries performed in United States per year [3-5]. Despite recent advances in technology, which have significantly improved cataract-related visual impairments [6-8], severe cataract-related disorders have significant negative effects on patients' quality of life, especially their mental health $[9,10]$. It has been previously demonstrated that cataract-related visual impairments are clearly associated with depression in elderly people [10, 11], which is further associated with increased risk of cognitive impairment, disability, and mortality [12-14]. The results of an Australian study showed that $43.4 \%$ of elderly people had eye diseases [15]. In addition, it has been shown that depressive symptoms are more common in cataract patients than in patients with other eye diseases [15]. In Iran, although studies in this field are very limited, the results of a study showed that $45.7 \%$ of cataract patients had moderate to severe levels of depression [16].

Meanwhile, cataract surgery seems to be effective in treating depression. Many study have revealed that the symptoms of depression can significantly reduce after a cataract surgery [17-19], however, some studies have reported conflicting results. According to a Japanese study, no statistical significance was found in the reduction of depression symptoms in elderly patients after cataract surgery [20]. Additionally, the results of a study in the United States showed that vision improvement after cataract surgery in elderly patients had no significant effect on reducing depression symptoms [21]. Considering the importance of potential negative psychosocial consequences of cataract and conflicting available evidence regarding the effect of cataract surgery on depressive symptom in older people, we aimed to evaluate the impact of cataract surgery on depression among elderly patients.

\section{Materials and Methods}

\subsection{Study design and sample}

In this case-control study, a total of 68 cataract patients and 45 elderly patients 60 years of age and older) without cataracts who were admitted to Buali-Sina Hospital in Sari, Mazandaran, Iran were evaluated. Data were collected using purposeful sampling. The case group consisted of 36 patients with cataract surgery (19 patients with bilateral 
and 17 with unilateral cataract) and 32 cataract patients without surgery (15 patients with bilateral and 17 with unilateral cataract). In addition, the control group consisted of 45 elderly patients without cataract disease.

\subsection{Inclusion and exclusion criteria}

The inclusion criteria were patients with unilateral or bilateral age-related cataracts, diagnosed by an ophthalmologist, aged $\geq 60$ years, and a visual acuity of $<0.4$ with glasses. Additionally, patients with neurological diseases such as Alzheimer's, Parkinson's, or history of coronary heart disease, traumatic cataracts, and advanced eye diseases such as retinal detachment, strabismus, macular degeneration, diabetic retinopathy, and glaucoma were excluded.

\subsection{Data collection}

Data were collected using two questionnaires, one including demographic and clinical characteristics of the patients (such as age, sex, weight, medication consumption, supplemental health insurance, vehicle, occupation, underlying diseases, visual acuity, and presence of refractive error), and other being the Hospital Anxiety and Depression Scale (HADS). The HADS questionnaire was designed to assess the severity of depressive and anxiety symptoms in patients [22]. Validity and reliability of this scale was assessed previously and was good. This self-reporting scale consists of 14 items, with subscales of anxiety (7 items) and depression (7 items), with scores ranging from 0 to 21. Each item consists of a 4-point Likert scale, from a score of 0 to 3. Scores between 0 and 7; 8 and 10; and 11 and 21 were considered as normal state, mild, and severe depression, respectively [23]. All patients were evaluated by an ophthalmologist on the day of admission. Snellen chart, with and without glasses, was used to evaluate the visual acuity of the patients. Also, presence of refractive errors was assessed using retinoscopy. After eye examinations by an optometrist, the HADS questionnaire was completed by participants. During surgery, an intraocular lens was implanted in all patients in the case group. Four months after the cataract surgery, visual acuity and refractive errors were measured and, if necessary, corrective glasses were prescribed. Also, the HADS questionnaire was completed again by patients. All participants in the control group completed the HADS questionnaire during the study period and their visual acuity was measured using a Snellen chart with and without glasses. 


\subsection{Statistical analysis}

Data were analyzed using the Statistical Package for the Social Sciences (SPSS) software (version 16.0, Chicago, IL, USA). The mean (standard deviation) and the number (percentage) were used to evaluate quantitative and qualitative variables, respectively. The $t$-test and ANOVA were used to evaluate the main variables of the study. $P$-value $<0.05$ was considered as statistically significant.

\section{Results}

A total of 113 patients, including 68 cataract patients and 45 elderly patients without cataract, were included in the present study. Of the patients with cataract, $42.6 \%$ were male and $51.5 \%$ were aged $66-70$ years (the mean and standard deviation of ages in patients with and without cataract was $69.12 \pm 1.83$ and $68.51 \pm 2.21$ years, respectively; $P=0.412$ ). Of the patients without cataracts, $57.8 \%$ were male and $68.9 \%$ were aged 61 70 years. Table 1 presents the demographic characteristics of patients with and without cataract.

Based on the results of the present study, there was a statistically significant difference between the mean depression scores in patients without cataract (3.28), patients with cataract surgery (3.74), and patients without cataract surgery $(5.60)(P=0.024)$. On the other hand, there was no statistically significant difference between the mean score of depression in patients with cataract surgery and patients without cataract $(P=0.582)$

The mean posttest depression score of bilateral cataract patients who had surgery in one eye (3.26) was lower than the mean pretest of the same group before cataract surgery (7.63) $(P<0.001)$. However, the mean posttest depression of unilateral atacract patients who had eye surgery (4.31) was lower than the mean pretest of the same group before cataract surgery (6.50) $(P=0.005)$. There was no statistically significant difference between the mean depression of unilateral cataract patients who had eye surgery (4.31) and bilateral cataract patients who had surgery in one eye (3.26) $(P=0.356)$. Moreover, there was a statistically significant difference between the mean depression of patients with cataract surgery (3.74) and patients without cataract surgery $(5.60)(P=0.187)$ (Table 2).

There was a statistically significant difference between the mean depression of patients without cataract surgery (5.60) and patients without cataract (3.28) $(P=0.031)$. However, there was no statistically significant difference between the mean depression of patients without cataract in the pretest (2.95) and the posttest (3.28) stages $(P=$ 
TABLE 1: Demographic characteristics of patients with and without cataract $(n=113)$.

\begin{tabular}{l} 
Variables \\
Age (yr) \\
Sex \\
Underlying diseases \\
\hline Medication consumption \\
\hline Supplemental health insurance \\
\hline Vehicle \\
\hline Occupation \\
\hline Weight (kg) \\
\hline
\end{tabular}

\begin{tabular}{|c|}
\hline $60-65$ \\
\hline $66-70$ \\
\hline$>70$ \\
\hline Male \\
\hline Female \\
\hline None \\
\hline Blood pressure \\
\hline Diabetic \\
\hline Yes \\
\hline No \\
\hline Yes \\
\hline No \\
\hline Yes \\
\hline No \\
\hline Freelancer \\
\hline Retired \\
\hline $61-70$ \\
\hline Unemployed/ housewife \\
\hline 60 \\
\hline 60 \\
\hline 60
\end{tabular}

\begin{tabular}{|c|c|}
\hline \multicolumn{3}{|c|}{ Group } \\
\hline With cataract & Without cataract \\
\hline $\mathbf{n}=\mathbf{6 8})$ & $\mathbf{( n = 4 5 )}$ \\
\hline $7(10.3)$ & $10(22.2)$ \\
\hline $35(51.5)$ & $31(68.9)$ \\
\hline $26(38.2)$ & $4(8.9)$ \\
\hline $29(42.6)$ & $26(57.8)$ \\
\hline $39(57.4)$ & $19(42.2)$ \\
\hline $47(69.1)$ & $33(73.4)$ \\
\hline $14(20.6)$ & $8(17.8)$ \\
\hline $7(10.3)$ & $4(8.9)$ \\
\hline $50(73.5)$ & $35(77.8)$ \\
\hline $18(26.5)$ & $10(22.2)$ \\
\hline $38(55.9)$ & $39(86.7)$ \\
\hline $30(44.1)$ & $6(13.3)$ \\
\hline $14(31.1)$ & $31(68.9)$ \\
\hline $53(77.9)$ & $15(22.1)$ \\
\hline $17(25)$ & $4(8.9)$ \\
\hline $13(19.1)$ & $27(60)$ \\
\hline $38(55.9)$ & $14(31.1)$ \\
\hline $36(52.9)$ & $9(20)$ \\
\hline $14(20.6)$ & $12(26.7)$ \\
\hline $18(26.5)$ & $24(53.3)$ \\
\hline
\end{tabular}

Data are presented as number (\%)

TABLE 2: Comparison of the severity of depression in patients with and without cataract surgery $(n=68)$

\begin{tabular}{|c|c|c|c|c|}
\hline & Mean & Standard Deviation & Degrees of freedom & P-value \\
\hline Patients with cataract surgery & 3.74 & 2.93 & 66 & 0.018 \\
\hline Patients without cataract surgery & 5.60 & 5.12 & & \\
\hline
\end{tabular}

0.284). In addition, there was a statistically significant difference between the mean depression score after cataract surgery in men (1.70) and women (4.94) $(P<0.001)$. However, based on the results of the present study, there was no statistically significant association between the mean depression score and age $(P=0.914)$. Visual function improved in all bilateral cataract patients who had surgery in one eye. Also, the results of this study showed that in all unilateral cataract patients who had eye surgery, visual function in the left and right eyes improved (Table 3). 
TABLE 3: Visual acuity of unilateral and bilateral cataract patients after surgery.

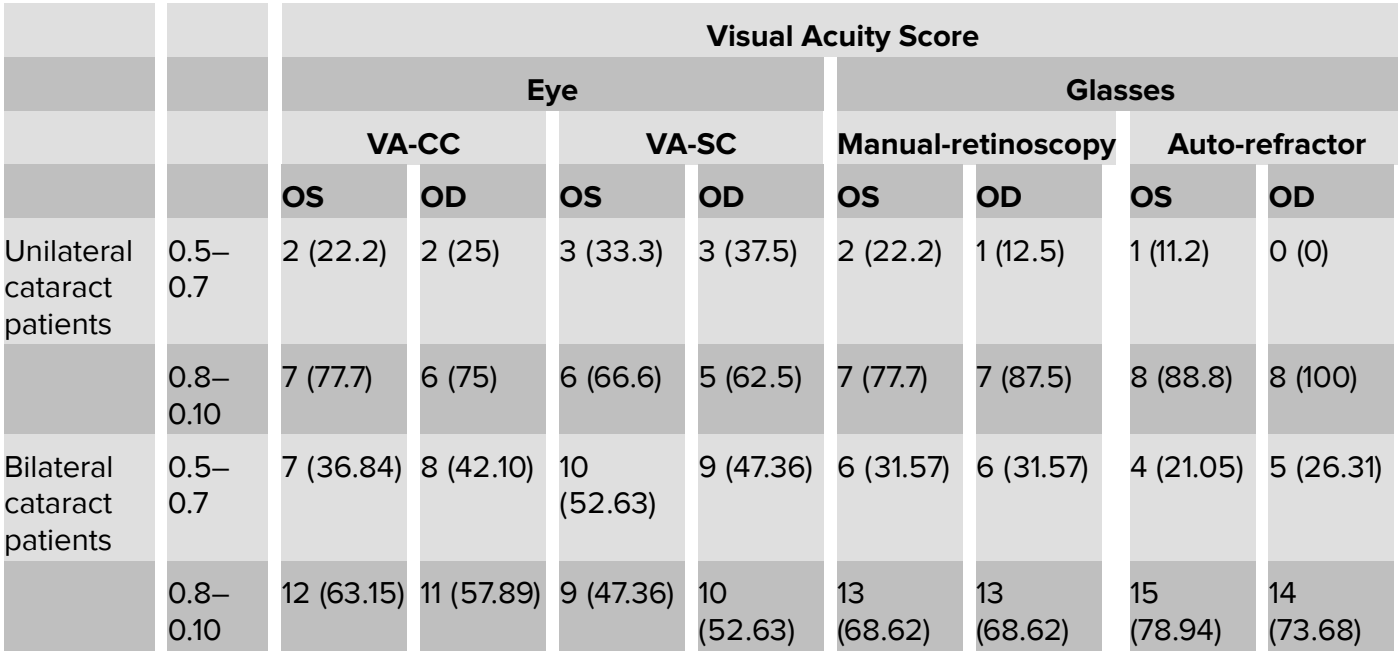

Data are presented as number (\%). OD: right eye; OS: left eye; VA-CC: visual acuity-with correction; VA-SC: visual acuity-without correction.

\section{Discussion}

The results of the present study showed that cataract aggravates depression in elderly patients. However, cataract surgery in each eye can reduce the symptoms of depression. In addition, patients without cataract surgery suffer more from depression than normal people. Moreover, a statistically significant relationship was observed between patients' gender and depression and cataract surgery. In addition, depression symptoms were significantly lower in men than in women who had cataract surgery. Visual function improved in all patients with bilateral cataract surgery.

Cataract disease is associated with an increased risk of mental disorders, such as depression and psychological distress, it is usually associated with reduced quality of patient's [24]. This finding is consistent with the results of studies from Vietnam [25, 26], Iran [18], Australia [10], and a joint study in Kenya, the Philippines, and Bangladesh [24]. On the other hand, contrary to the findings of the present study, the results of a study in the United States [21] showed that cataract surgery has no significant effect on reducing patients' depression symptoms. Similarly, the results of an Australian study [27] also showed that cataract surgery increases vision-related quality of life, however, its effect on depressive symptoms was unknown. One possible explanation for this inconsistency may be the higher severity of depression in patients before surgery in the present study [25]. On the other hand, in the present study, the symptoms of depression in men were significantly lower than in women who had cataract surgery, which was inconsistent with previous studies in Canada [28, 29], the United States [30], and Vietnam [25]. It has been previously shown that the prevalence of depression in Iranian female patients is higher 
than male patients [31,32]. Therefore, higher severity of depression in women than men can be the reason for the lower severity of depression in women after cataract surgery [25]. It has been shown that bilateral cataract surgery is associated with improved visual function and cognitive function after surgery [18]. Therefore, the negative effect of visual impairment on cognitive status in these patients can be attributed to cataracts [33-36], and surgical intervention can improve patients' visual and cognitive impairment [37, 38]. The results of Jefferis et al.'s study revealed that visual and cognitive function improved after bilateral cataract surgery [39]. Inconsistent with this finding, a study in the United States [40] showed an improvement of cognitive function and vision in older patients without cataract surgery.

\section{Conclusion}

In conclusion, it seems that cataract can cause and exacerbates depression, and surgery can reduce the severity of depression in elderly patients. Moreover, in all patients who had unilateral cataract and were operated, increased vision was observed in both the right and left eyes. However, there is still insufficient evidence for cognitive improvement and reducing depression in patients after cataract surgery. Future studies are needed to validate our findings in different study population.

\section{Limitations}

This study has some limitations. Our study was observational and correlational in nature, and hence causality could not be established. There is a need for other well-designed cohort studies, preferably with longer duration of follow-up, to confirm the results of the present study. Additionally, only those patients who were referred to a teaching hospital in north of Iran were evaluated in this study, therefore, generalizability of the findings to other populations in different geographic regions is unclear.

\section{Acknowledgments}

None. 


\section{Ethical Considerations}

This study was approved by the university ethics committee. The objective of the study was explained to all participants and a written informed consent was obtained from all prior to the study. Furthermore, for the confidentiality of patient information, participants' names were removed from the questionnaires.

\section{Competing Interests}

The authors have no conflicts of interest to declare for this study.

\section{Availability of Data and Material}

All relevant data of this study are available to any interested researchers upon reasonable request to the corresponding author.

\section{Funding}

This study has been financially supported by the Deputy of Research and Technology, Mazandaran University of Medical Sciences, Sari, Iran.

\section{References}

[1] Ackland, P., Resnikoff, S., and Bourne, R. (2017). World blindness and visual impairment: despite many successes, the problem is growing. Community Eye Health Journal, vol. 30, no. 100, pp. 71-73.

[2] Liu, Y. C., Wilkins, M., Kim, T., et al. (2017). Cataracts. The Lancet, vol. 5, no. 390, pp. 600-612.

[3] Schein, O. D., Cassard, S. D., Tielsch, J. M., et al. (2012). Cataract surgery among Medicare beneficiaries. Ophthalmic Epidemiology, vol. 19, no. 5, pp. 257-264.

[4] Allen, D. and Vasavada, A. (2006) Cataract and surgery for cataract. The BMJ, vol. 333, no. 7559, pp. 128-132.

[5] Tabin, G., Chen, M., and Espandar, L. (2008). Cataract surgery for the developing world. Current Opinion in Ophthalmology, vol. 19, no. 1, pp. 55-59.

[6] Davis, G. (2016). The evolution of cataract surgery. Missouri Medicine, vol. 113, no. 1, pp. 58-62. 
[7] Ashwin, P. T., Shah, S., Wolffsohn, J. S. (2009). Advances in cataract surgery. Clinical and Experimental Optometry, vol. 92, no. 4, pp. 333-342.

[8] Ho, J. W. and Afshari, N. A. (2015). Advances in cataract surgery: preserving the corneal endothelium. Current Opinion in Ophthalmology, vol. 26, no. 1, pp. 22-27.

[9] Skalicky, S. E., Martin, K. R., Fenwick, E., et al. (2015). Cataract and quality of life in patients with glaucoma. Clinical \& Experimental Ophthalmology, vol. 43, no. 4, pp. 335-341.

[10] Palagyi, A., Rogers, K., Meuleners, L., et al. (2016). Depressive symptoms in older adults awaiting cataract surgery. Clinical \& Experimental Ophthalmology, vol. 44, no. 9, pp. 789-796.

[11] Safiri, S. and Ayubi, E. (2017). Depressive symptoms in older adults awaiting cataract surgery: methodological and statistical issues. Clinical \& Experimental Ophthalmology, vol. 45, no. 8, pp. 840-841.

[12] Alexopoulos, G. S. (2005). Depression in the elderly. The Lancet, vol. 365, no. 9475, pp. 1961-1970.

[13] Barua, A., Ghosh, M. K., Kar, N., et al. (2011). Prevalence of depressive disorders in the elderly. Annals of Saudi Medicine, vol. 31, no. 6, pp. 620-624.

[14] Fiske, A., Wetherell, J. L., and Gatz, M. (2009). Depression in older adults. Annual Review of Clinical Psychology, vol. 5, pp. 363-389.

[15] Eramudugolla, R., Wood, J., and Anstey, K. J. (2013). Co-morbidity of depression and anxiety in common age-related eye diseases: a population-based study of 662 adults. Frontiers in Aging Neuroscience, vol. 5, no. 56, pp. 1-9.

[16] Samarai, V. and Aidenlou, N. S. (2016). A survey on the level of depression in cataract patients hospitalized in urmia. Research Journal of Medical Sciences, vol. 10, no. 4, pp. 282-286.

[17] Pellegrini, M., Bernabei, F., Schiavi, C., et al. (2020). Impact of cataract surgery on depression and cognitive function: systematic review and meta-analysis. Clinical \& Experimental Ophthalmology, vol. 48, no. 5, pp. 593-601.

[18] Kheirkhah, F., Roustaei, G., Mohebbi Abivardi, E., et al. (2018). Improvement in cognitive status and depressive symptoms three months after cataract surgery. Caspian Journal of Internal Medicine, vol. 9, no, 4, pp. 386-392.

[19] Miyata, K., Yoshikawa, T., Morikawa, M., et al. (2018). Effect of cataract surgery on cognitive function in elderly: results of Fujiwara-kyo Eye Study. PLoS One, vol. 13, no. 2, p. e0192677. 
[20] Ishii, K., Kabata, T., and Oshika, T. (2008). The impact of cataract surgery on cognitive impairment and depressive mental status in elderly patients. American Journal of Ophthalmology, vol. 146, no. 3, pp. 404-409.

[21] McGwin Jr., G., Li, J., McNeal, S., et al. (2003). The impact of cataract surgery on depression among older adults. Ophthalmic Epidemiology, vol. 10, no. 5, pp. 303313.

[22] Zigmond, A. S. and Snaith, R. P. (1983). The Hospital Anxiety and Depression Scale. Acta Psychiatrica Scandinavica, vol. 67, no. 6, pp. 361-370.

[23] Montazeri, A., Vahdaninia, M., Ebrahimi, M., et al. (2003). The Hospital Anxiety and Depression Scale (HADS): translation and validation study of the Iranian version. Health and Quality of Life Outcomes, vol. 1, no. 14, pp.1-5.

[24] Polack, S., Eusebio, C., Mathenge, W., et al. (2010). The impact of cataract surgery on health related quality of life in Kenya, the Philippines, and Bangladesh. Ophthalmic Epidemiology, vol. 17, no. 6, pp. 387-399.

[25] To, K. G., Meuleners, L. B., Fraser, M. L., et al. (2014). The impact of cataract surgery on depressive symptoms for bilateral cataract patients in Ho Chi Minh City, Vietnam. International Psychogeriatrics, vol. 26, no. 2, pp. 307-313.

[26] Berle, D., Steel, Z., Essue, B. M., et al. (2017). Multisite prospective investigation of psychological outcomes following cataract surgery in Vietnam. BMJ Glob Health, vol. 2, no. 1, p. e000162.

[27] Meuleners, L. B., Agramunt, S., Ng, J. Q., et al. (2015). The Cataract Extraction and Driving Ability Research Study Protocol: characterisation of deficits in driving performance and self-regulation among older drivers with bilateral cataract. Injury Prevention, vol. 21, no. 6, pp. 424-429.

[28] Franks, F. and Faux, S. A. (1990). Depression, stress, mastery, and social resources in four ethnocultural women's groups. Research in Nursing \& Health, vol. 13, no. 5, pp. 283-292.

[29] Freeman, E. E., Gresset, J., Djafari, F., et al. (2009). Cataract-related vision loss and depression in a cohort of patients awaiting cataract surgery. Canadian Journal of Ophthalmology, vol. 44, no. 2, pp. 171-176.

[30] Owsley, C., McGwin Jr., G., Scilley, K., et al. (2007). Impact of cataract surgery on health-related quality of life in nursing home residents. British Journal of Ophthalmology, vol. 91, no. 10, pp. 1359-1363.

[31] Ghaemmohamadi, M. S., Behzadifar, M., Ghashghaee, A., et al. (2018). Prevalence of depression in cardiovascular patients in Iran: a systematic review and meta-analysis from 2000 to 2017. Journal of Affective Disorders, vol. 227, pp. 149-155. 
[32] Ghadirian, L. and Sayarifard, A. (2019). Depression literacy in urban and suburban residents of Tehran, the capital of Iran; recognition, help seeking and stigmatizing attitude and the predicting factors. International Journal of Preventive Medicine, vol. 10, no. 134, pp. 1-6.

[33] Choi, H. G., Lee, M. J., and Lee, S. M. (2018). Visual impairment and risk of depression: a longitudinal follow-up study using a national sample cohort. Scientific Reports, vol. 8, no. 1, p. 2083.

[34] Ribeiro, M. V., Hasten-Reiter Júnior, H. N., Ribeiro, E. A., et al. (2015). Association between visual impairment and depression in the elderly: a systematic review. Arquivos Brasileiros de Oftalmologia, vol. 78, no. 3, pp. 197-201.

[35] Swenor, B. K., Wang, J., Varadaraj, V., et al. (2019). Vision impairment and cognitive outcomes in older adults: the Health ABC Study. The Journals of Gerontology. Series A, Biological Sciences, vol. 74, no. 9, pp. 1454-1460.

[36] Heesterbeek, T. J., van der Aa, H. P. A., van Rens, G., et al. (2017). The incidence and predictors of depressive and anxiety symptoms in older adults with vision impairment: a longitudinal prospective cohort study. Ophthalmic and Physiological Optics, vol. 37, no. 4, pp. 385-398.

[37] Jefferis, J. M., Clarke, M. P., and Taylor, J. P. (2015). Effect of cataract surgery on cognition, mood, and visual hallucinations in older adults. Journal of Cataract \& Refractive Surgery, vol. 41, no. 6, pp. 1241-1247.

[38] Miyata, K., Obayashi, K., Saeki, K., et al. (2016). Higher cognitive function in elderly individuals with previous cataract surgery: cross-sectional association independent of visual acuity in the HEIJO-KYO cohort. Rejuvenation Research, vol. 19, no. 3, pp. 239-243.

[39] Jefferis, J. M., Taylor, J. P., Clarke, M. P. (2015). Does cognitive impairment influence outcomes from cataract surgery? Results from a 1-year follow-up cohort study. British Journal of Ophthalmology, vol. 99, no. 3, pp. 412-417.

[40] Hall, T. A., McGwin Jr., G., and Owsley, C. (2005). Effect of cataract surgery on cognitive function in older adults. Journal of the American Geriatrics Society, vol. 53 , no. 12 , pp. $2140-2144$. 